\title{
Joint Optimization of Smart Antenna System and Wireless Mesh Network
}

\author{
Yulong Chen and Ning Xie \\ College of Information Engineering Shenzhen University Shenzhen, Guangdong 518060, China. \\ Email: ningxie@szu.edu.cn
}

Keywords: Wireless Mesh Networks; smart antennas; OPNET; MATLAB

\begin{abstract}
Various network architectures are evolved into the next generation wireless system to provide better services, such as wireless mesh networks (WMNs). However, single radio node mesh operating on a single channel suffers from capacity limiting. Multiple radio nodes mesh can obviously improve the network capacity, but the network is susceptible to spatial interference. Smart antennas are therefore employed to overcome such problem in this paper. A multiple-beam with circular RTS scheme is proposed to solve the "deafness" problem, which includes two parts: 1) expanding single interface to multi-interfaces in a node for simultaneous communicating with multiple nodes; 2) employing the circular RTS scheme for quick establishing communication with multiple nodes. This scheme is simulated by OPNET 14.5 and MATLAB R2007a. Simulation results show that the proposed scheme not only improve the network throughput but also solve the deafness problem.
\end{abstract}

\section{INTRODUCTION}

The existing WMNs mainly utilizes omni-directional antenna or directive antenna, however there exist some obvious drawbacks. On one hand, the application of omni-directional antenna in WMNs results in less coverage, which will cause more hops and network delay. Furthermore, since omnidirectional antenna radiates uniformly in all directions, which results in a considerable amount of spatial interference and reduces throughput[4]. On the other hand, compared to omni-directional antenna, directive antenna can expand coverage and enhance the throughput[5] but it lacks of nulling control and controlling the level of sidelobes, which also cause some problem, such as deafness problem.

Thanks to smart antenna system, it can form an adaptive beam according to the desired signal angle and interfering signal angles[6, 7], and it can significantly increase the coverage and reduce network relay burden[8]. A multiple-beam with circular RTS scheme is proposed to solve the "deafness" problem, which includes two parts: 1) expanding single interface to multi-interfaces in a node for simultaneous communicating with multiple nodes; 2) employing the circular RTS scheme for quick establishing communication with multiple nodes. In order to analyze the effect of smart antenna system on the upper layers (specifically, data link and network layers) of the communication system, we develop an antenna controller using OPNET Modeler[9]. The antenna controller interfaces with the upper layer and physical layer to form the antenna gain pattern adaptively. By integrating MATLAB and OPNET, we are able to reuse any adaptive beamforming algorithms.

\section{THE MULTIPLE-BEAM WITH CIRCULAR RTS SCHEME}

\section{Employing the multiple-beam with circular RTS scheme to solve the "deafness" problem}

Deafness problem is very common when using directional antenna or smart antennas[10]. Smart antennas can't send/receive packet in the side lobe because of the power gain is very small. Thus, it is "deafness" in the side lobe direction. In Fig.1., for example. node A、 node B and node C all use directional or smart antennas, and each node only has a main lobe. Node A and node B is in each other's coverage range, so they can establish communication. Node B is in node C's coverage range, but node C isn't in node B' coverage range. Thus, node B can't receive any massage from node C. 


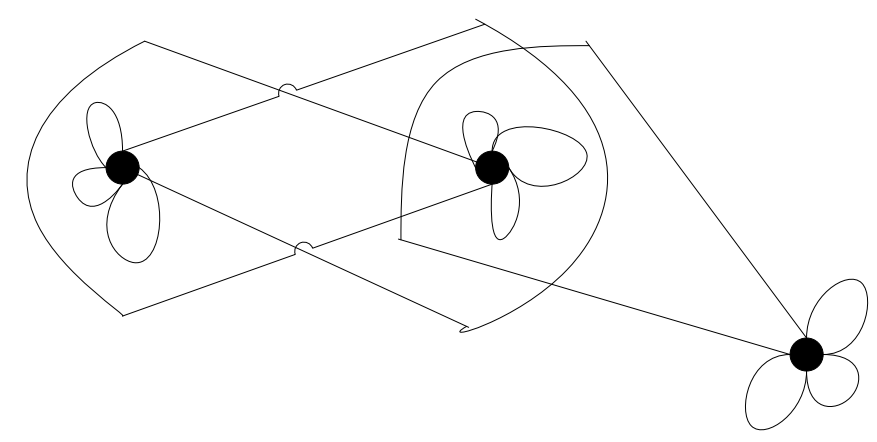

Fig. 1. "Deafness” problem of smart antennas

The existing wireless mesh networks mainly utilize omni-directional antenna. However, the omnidirectional antenna limits the coverage[11]. Fig. 2 shows the coverage range of smart antennas and omni-directional antenna[12]. In order to solve the "deafness" problem when using smart antennas, we propose a multiple-beam with circular RTS scheme, shown in Fig. 3. At first, a single interface in a node is expanded to multi-interfaces. Each interface adaptively form beam according to the desired angle and interfering angles. Thus, each node has multiple main lobes. We suppose that a node has two interfaces and the area around it is divided into eight sectors. We employ circular RTS scheme to scan all the sectors. The transmitter starts transmitting its RTS from sector 1 on. It continues to send RTS until the transmission of RTS covers all the area around the transmitter. After circular send RTS, the node already had the location information of its neighbor nodes.

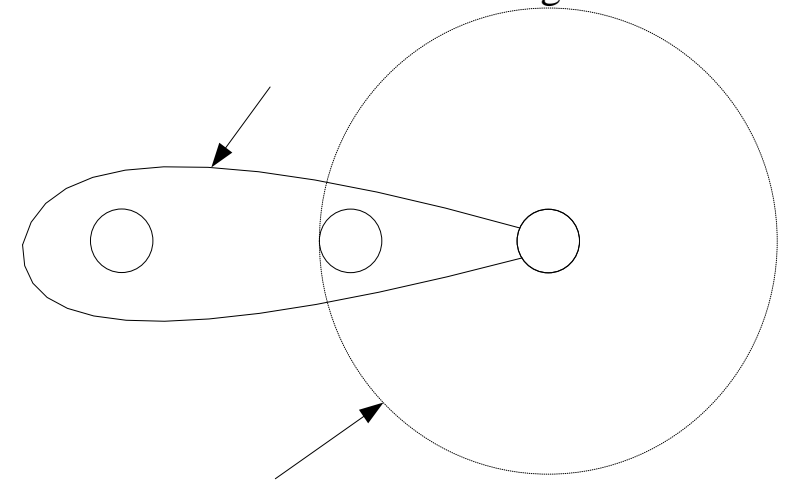

Fig. 2. The coverage range of omni-directional transmission and directional transmission

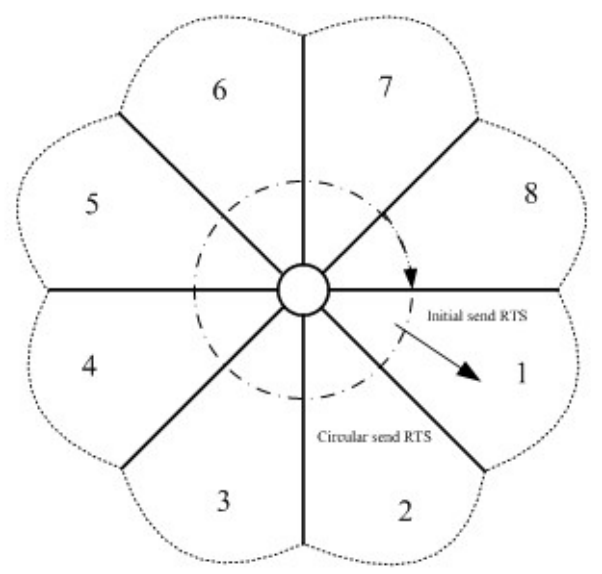

Fig. 3. A node with circular send RTS 


\section{SINGLE HOP SIMULATION}

This section includes two parts: single hop simulation and multi-hop simulation. A single hop network model is constructed to compare the performances of omni-directional antenna, directional antenna and smart antennas. Another single hop network is constructed to examine if the multiplebeam with circular RTS scheme can solve the "deafness" problem.

\section{Antenna performance comparison}

\section{Simulation scenario}

This scenario is a simple wireless network with a mobile jammer node and two stationary communications nodes. The jammer node and transmitter node use omni-directional antenna. Receiver node use omni-directional antenna, directional antenna and smart antennas.

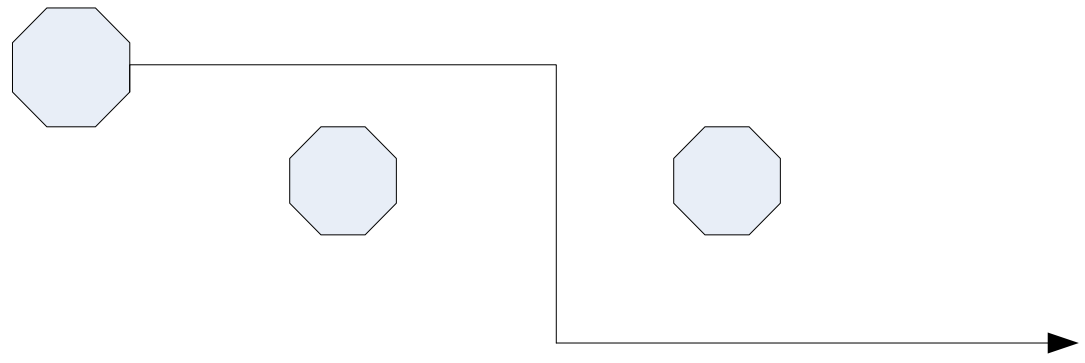

Fig. 4. Simulation scenario

The jammer node and transmitter node all send the packets to the receiver node at the same time. The transmitter node and receiver node are stationary, while the jammer node goes along the trajectory in and out of the radio range of the receiver node, increasing and decreasing interference at the receiver. Packets are generated using constant inter arrival times and constant packet size of 1024 bits.

During simulation, the interfering angle change all the time. When the receiver node use smart antennas, the receive gain pattern will change follow by the interfering angle.

\section{Simulation result}
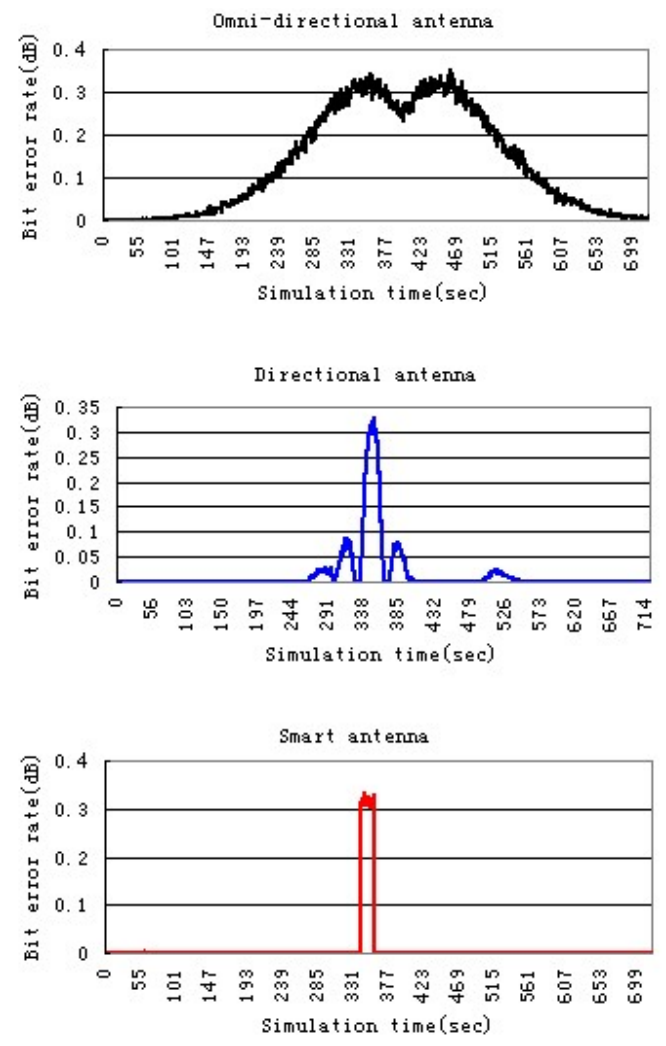

Fig. 5. The bit error rate of receiver 
During the simulation, collecting receiver node rx network performance factors, such as throughput, bit error rate and receiver power. Fig. 5 shows the receiver bit error rate, the receive bit error rate has a min value when receiver use smart antenna the receiver bit error rate is always zero except jammer node between transmitter node and receiver node. This is because the adaptive beam forming algorithm form null in the interfering angle, suppressing the interference signals. Since omni-directional antennas radiate uniformly in all directions, the receiver bit error rate goes up as the jammer node going close to the receiver node.

From Fig. 5, we can see that the bit error rate is inversely proportional to the throughput. When the bit error rate has a low value, throughput is high, when the bit error rate has a higher value, the throughput is lower, even zero, this is consistent with the theoretical analysis.

\section{Solve "deafness" problem}

\section{Simulation scenario}

The node A and node Call send packets to node B at the same time. The packet is generated by the constant interarrival time 1 and constant packet size of 1024 bits. The simulation during time is 30 minute. There have two cases in this scenario. The one is that the receiver node $\mathrm{B}$ only has a beam and the main lobe toward to node $\mathrm{A}$. The other one is that the receiver node $\mathrm{B}$ has two beams and main lobes toward to node Aand node $\mathrm{C}$ respectively with circular RTS scheme. In the first scenario, node B only has a beam, its main lobe aim at node A and its side lobe toward to node C. Thus, node B always can't receive any message from node C. In the other one scenario, the receiver node B has two interfaces and the area around it is divided into eight sectors. We employ circular RTS scheme to scan all the sectors. The transmitter starts transmitting its RTS from sector 1 on. It continues to send RTS until the transmission of RTS covers all the area around the transmitter. After circular send RTS, the node already had the location information of its neighbor nodes. Each interface adaptively form beam according to the location information of its neighbor nodes.

\section{Simulation results}

During the simulation, the bit error and throughput are collected by receiver node. Fig. 6 and Fig. 7 show the bit error and throughput of receiver node in the scenario of receiver node $\mathrm{B}$ only has a beam. From the figures we can observe that the bit error of link between node B and node A is 0 and the throughput is 1024 bits/sec. while the bit error of link between node B and node $\mathrm{C}$ is very high and the throughput is $0 \mathrm{bits} / \mathrm{sec}$. this is because that the main lobe of the receiver node $\mathrm{B}$ toward to node A, while the side lobe toward to node B. Thus node B can't receive packets from node C. In other word, node B is deafness to node C.

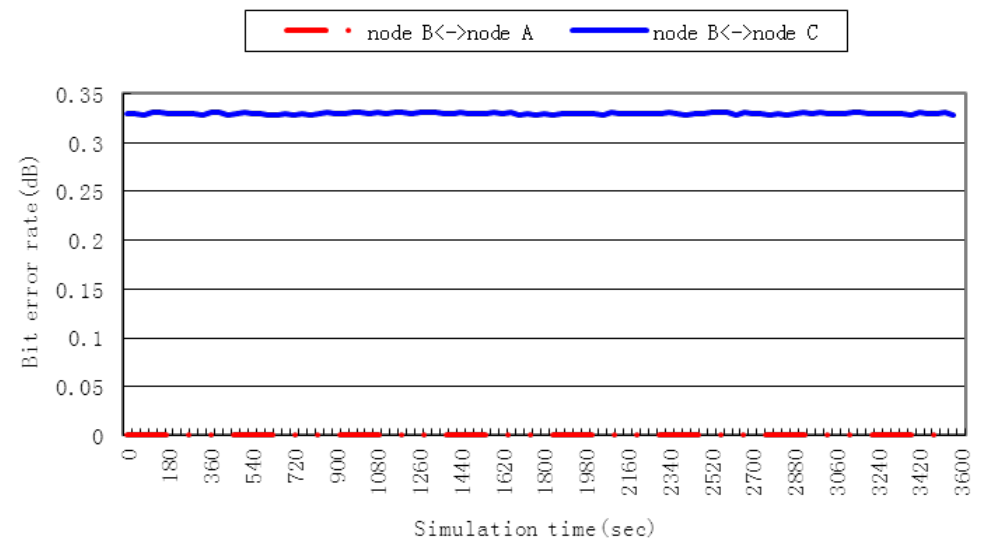

Fig. 6 . The bit error rate of receiver with a beam 


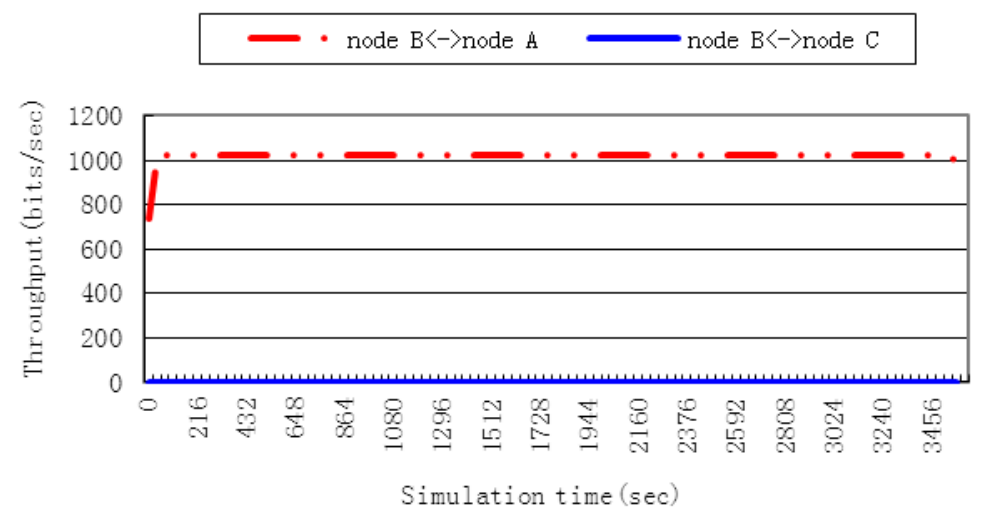

Fig. 7. The throughput of receiver with a beam

\section{MULTI-HOP SIMULATION}

In this section, two different multi-hop network topologies are constructed to analyze how the smart antenna affects the network performance. AODV routing protocol is employed in the multihop network. The AODV routing protocol is an on-demand distance vector routing protocol[13].

\section{Simulation scenario}

In this section, there are 16 nodes in the network. All the nodes employ the AODV routing protocol. The node in this topology also have two cases: one case is that all the nodes use omnidirectional antenna, the other case is that all the nodes use smart antennas. At the run time, the routing path, end to end delay and bit error are collected.

\section{Simulation results}

We record the all the routing paths during simulation. It is clear that the routing hop when nodes use smart is fewer than when nodes use omni-directional antenna.

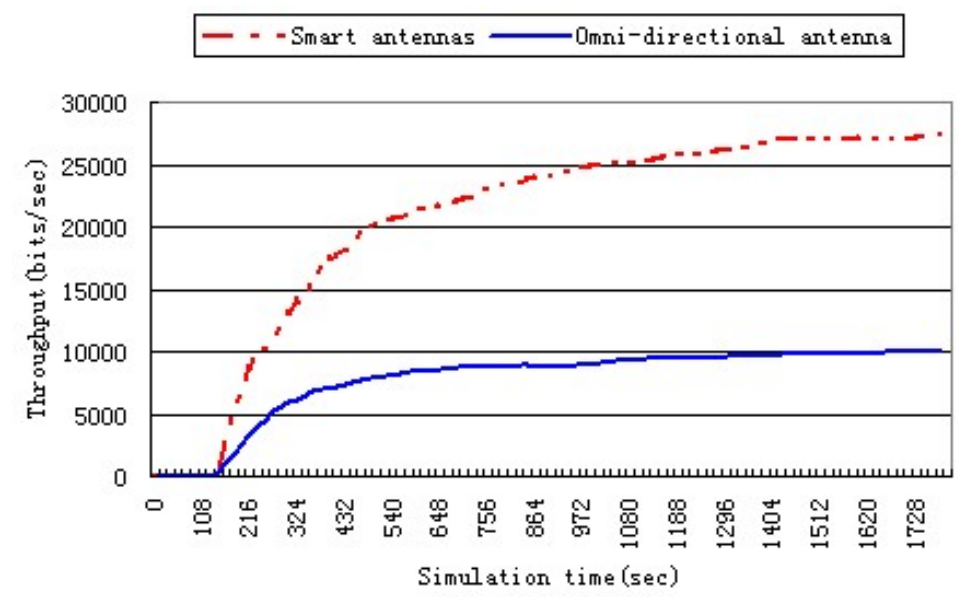

Fig. 8. The throughput of network

We can see that it can reduce the end to end delay when nodes use smart antennas, as Fig. 8 shown. Smart antennas expand the coverage range, thereby reduce the end to end delay. The adaptive beam of smart antennas forming can cancellation interference and improve the spatial reuse. It leads to improve the SNR. As shown in Fig. 8, the throughput of smart antennas is better than that of omni-directional antenna.

\section{CONCLUSION}

In this paper we have presented a methodology for implementing smart antennas and WMNs in OPNET. A multiple-beam with circular RTS scheme is proposed to solve the deafness problem. A simple network model is constructed to analyze the performances of various antenna architectures 
and the multiple-beam with circular RTS scheme. Simulation results show that such scheme can solve the deafness problem, and effectively reduce the routing hops, suppress interference and improve network throughput.

\section{ACKNOWLEDGEMENT}

This work was partially supported by Natural Science Foundations of China (No. 61001182), Natural Science Foundation of Guangdong, China (No.S2013010012227, No. 10451806001004788), Science and Technology innovation Programs of Colleges and Universities in Guangdong (No. 2013KJCX0160), Fundamental Research Programs of Shenzhen City ( No . J C Y J 20150324141 71169 0, No. JCY J 20130329105415965 ).

\section{REFERENCES}

[1]Akyildiz, I., X. Wang, and X.W. Wang, Wireless mesh networks: A survey. Elsevier Journal on Computer Networks, 2005. 47: p. 445 - 487.

[2]Akyildiz, I. and X. Wang, A survey on wireless mesh network. IEEE Communication Magazine, 2005. 43: p. 23 - 30.

[3]Piggin, P. and P. B. Lewis, Whitehead mesh networks in fixed broadband wireless access: multipoint enhancements for the 802.16 standard. IEEE 802.16 presentation slides, July 2003.

[4]Muthaiah, S.N., et al., Design of High Throughput Scheduled Mesh Networks: A Case for Directional Antennas. Global Telecommunications Conference, 2007. GLOBECOM '07. IEEE, 2007: p. 5080-5085.

[5]Spyropoulos, A. and C.S. Raghavendra, Energy efficient communications in ad hoc networks using directional antenna. IEEE Annual Conference on Computer Communications (INFOCOM), 2002: p. 220-228.

[6]Liberti, J.C. and T. Rappaport, eds. Smart Antennas for Wireless Communications: IS 95 and Third Generation CDMA Applications. 1999, Prentice Hall PTR: Upper Saddle River, New Jersey.

[7]Bellofiore, S., et al., Smart antenna system analysis, integration and performance for mobile ad hoc networks (MANETs). IEEE Transactions on Antennas and Propagation, 2002. 5: p. 571-58.

[8]Spyropoulos, A. and C.S. Raghavendra, Asymptotic capacity bounds for ad hoc networks revisited: the directional and smart antenna cases. IEEE Global Telecommunications Conference (GLOBECOM), 2003: p. 1216-1220.

[9]Chang, X. Network Simulations with OPNET. in Proceedings of the 1999 Winter Simulation Conference. 1999. New York.

[10]Choudhury, R.R. and N.H. Vaidya. Deafness: A Mac problem in ad hoc networks when using directional antennas. in Proc. of the 12th IEEE International Conference on Network Protocols (ICNP'04). 2004. Berlin, Germany.

[11]Mundarath, J., P. Ramanathan, and B.V. Veen, Exploiting spatial multiplexing and reuse in multi-antenna wireless ad hoc networks. Ad Hoc Networks, 2009. 7(2): p. 281 - 293.

[12]Korakis, T., G. Jakllari, and L. Tassiulas. A MAC protocol for full exploitation of directional antennas in ad-hoc wireless networks. in Proc. ACM Mobile Ad Hoc Networking and Computing (MobiHoc). June 2003. Maryland, USA.

[13]Hossain, E. and K. Leung, Wireless mesh networks : architectures and protocols 2008, New York Springer. 173-293. 